\title{
A comparison theorem for functional differential equations
}

\section{Athanassios G. Kartsatos and Hiroshi Onose}

\begin{abstract}
In this paper, we study the oscillation of $n$ th-order differential equations. Recently, Atkinson and the present authers studied (separately) the comparison properties of differential inequalities. Kartsatos treated the $n$ th-order ordinary case and proposed several open problems.

The purpose of this paper is to answer one of them in the affirmative concerning more general functional differential equations. The result is that if under several conditions, the equation
\end{abstract}

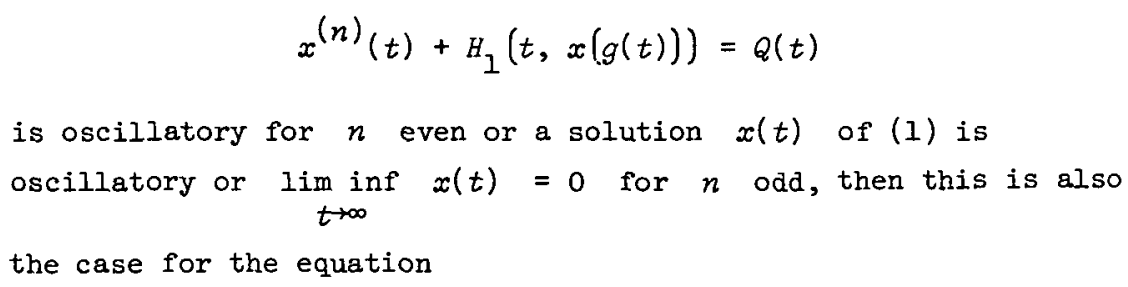$$
x^{(n)}(t)+H_{1}(t, x(g(t)))=Q(t)
$$

$$
x^{(n)}(t)+H_{2}(t, x(g(t)))=Q(t) .
$$

\section{Introduction}

Recently, Atkinson [1] and the present authors [2], [3], [5] studied the comparison properties of differential inequalities. Kartsatos [2], [3], treated the $n$ th-order ordinary case and proposed several open problems.

Received 28 January 1976. 
The purpose of this paper is to answer one of them in the affirmative concerning more general functional differential equations.

Consider the functional differential equations

$$
x^{(n)}(t)+H_{i}(t, x(g(t)))=Q(t) \quad(i=1,2) .
$$

In what follows, $R=(-\infty, \infty), R_{+}=[0, \infty)$. The functions $H_{i}(t, x)$ ( $i=1,2)$ will be defined and continuous on $R_{+} \times R$ with values in $R$. By a solution of equation (*;, we mean any function $x \in C^{n}\left[t_{x}, \infty\right)$, which satisfies (*) for all $t \in\left[t_{x}, \infty\right)$. Here $t_{x}$ depends on the solution $x(t)$. A solution $x(t)$ is said to be oscillatory if it has an unbounded set of zeros in its interval of definition $\left[t_{x}, \infty\right)$. If all solutions of (*) are oscillatory, then equation (*) is said to be oscillatory.

\section{The result}

THEOREM. Let the functions $H_{i}(t, u), i=1,2$ be defined on $R_{+} \times R$, increasing with respect to $u$, and such that $u H_{i}(t, u)>0$ for $u \neq 0$. Let $P \in C^{n}\left[R_{+}, R\right], P^{(n)}(t) \equiv Q(t)$ for every $t \in R_{+}$, and $\lim _{t \rightarrow \infty} P(t)=0$. Let the function $g(t)$ be continuous on $R_{+}$and such that $t \rightarrow \infty$

$g(t) \leq t, \lim _{t \rightarrow \infty} g(t)=\infty$. Then if $P(t)$ is oscillatory and

$$
\begin{aligned}
& H_{1}(t, u) \leq H_{2}(t, u), \quad t \in R_{+}, u \geq 0, \\
& H_{1}(t, u) \geq H_{2}(t, u), \quad t \in R_{+}, u<0,
\end{aligned}
$$

and the equation

$$
x^{(n)}(t)+H_{1}(t, x(g(t)))=Q(t)
$$

is oscillatory for $n$ even or a solution $x(t)$ of (1) is oscillatory or lim inf $x(t)=0$ for $n$ odd, this is also the case for the equation $t \rightarrow \infty$

$$
x^{(n)}(t)+H_{2}(t, x(g(t)))=Q(t) .
$$


Proof. Let (2) be nonoscillatory for $n$ even and any solution $z(t)$ of (2) be nonoscillatory and $\lim _{t \rightarrow \infty}|z(t)|>0$ for $n$ odd. Assume that a solution $z(t)$ of equation (2) is positive for $t \geq T \geq t_{z}$. Then the function $u(t) \equiv z(t)-P(t)$ is an eventually positive solution of the equation

$$
u^{(n)}(t)+H_{2}(t, u(g(t))+P(g(t)))=0
$$

In fact, $u(g(t))+P(g(t))>0$ on $\left.\mid T_{1}, \infty\right)$ with $T_{1} \geq T$, implies $u^{(n)}<0$ on $\left[T_{1}, \infty\right)$. Consequently, $u(t)$ has to be eventually of constant sign. If $u(t)<0$ for all large $t$, then $P(t)>-u(t)>0$ for all large $t$, a contradiction to the oscillatory character of $P(t)$. Let $u(t)>0$ eventually. By $u^{(n)}(t)<0, u(t)>0$ and Kiguradge's Lemma [4], there exists an odd (even) integer with $0 \leq l \leq n-1$ for $n$ even (odd) such that

$$
\begin{gathered}
u^{(i)}(t)>0, i=0,1, \ldots, \tau, \\
(-1)^{2+i} u^{(i)}(t) \geq 0, i=\imath+1, \ldots, n, \text { for } t \geq T_{1} .
\end{gathered}
$$

Thus, in particular, $u(t)>0, u^{\prime}(t)>0$ for every $t \geq T_{1}$ if $n$ is even or odd, or, possibly, for $n$ odd, $u(t)>0, u^{\prime}(t)<0$ for every $t \geq T_{1}$. Let now $T_{1}$ be so large that we also have $|P(t)|<c<u\left(T_{1}\right)$ for all $t \geq T_{1}$, where we can take $c$ be a positive constant. Then we obtain

$$
u^{(n)}(t)+H_{1}(t, u(g(t))+P(g(t))) \leq u^{(n)}(t)+H_{2}(t, u(g(t))+P(g(t)))=0
$$

for every $t \geq T_{1}$.

Notice that $u(g(t))+P(g(t))>0$ for $t \geq T_{1}$. Consequently, the inequality

$$
u^{(n)}(t)+B_{1}(t, u(g(t))+P(g(t))) \leq 0
$$

has a solution $u(t)$ with the property that $u(t)>0, u^{\prime}(t)>0$ for 
$u^{\prime}(t)<0$ in some odd case). By repeated integration of (4), we obtain

(5) $u(t) \geq u\left(T_{1}\right)+\int_{T_{1}}^{t} \int_{T_{1}}^{s_{n-1}} \ldots \int_{T_{1}}^{s_{n-l+1}} \int_{s_{n-l}}^{\infty} \ldots$

$$
\begin{gathered}
\ldots \int_{s_{1}}^{\infty} H_{1}(t, u(g(t))+P(g(t))) d t d s_{1} \ldots d s_{n-1} \\
\geq c+\Psi(t, u(g(t))+P(g(t))), \quad\left(t \geq T_{1}\right),
\end{gathered}
$$

where $c=u\left(T_{1}\right)$ in case $u^{\prime}(t)>0$ and $c=u\left(T_{1}\right) / 2$ in case $u^{\prime}(t)<0$.

Now it is easy to show the existence of a positive solution to the integral equation

$$
v(t)=c+\Psi(t, v(g(t))+P(g(t))), \quad t \geq T_{1} .
$$

We define $v_{n}(t), n=0,1, \ldots$ such that

$$
\begin{aligned}
& v_{0}(t)=u(t) \quad \text { for } t \geq T \text {, } \\
& v_{n+1}(t)= \begin{cases}c+\Psi\left(t, v_{n}+P\right) & \text { for } t \geq T_{1} \\
c & \text { for } T \leq t \leq T_{1} .\end{cases}
\end{aligned}
$$

Then we see that $v_{n}(t)$ is well-defined and

$$
0<v_{n}(t)<u(t), c \leq v_{n+1}(t) \leq v_{n}(t) \text {. }
$$

If we put

$$
v(t)=\lim _{t \rightarrow \infty} v_{n}(t) \text { for every point } t \geq T_{1},
$$

then by $(7),(8)$, and Lebesgue's Theorem we have

$$
v(t)=c+\Psi(t, v(g(t))+P(g(t))) \text {, for all } t \geq T_{1} .
$$

If we differentiate (6) $n$ times, we obtain

$$
r^{(n)}(t)+H_{1}(t, r(g(t)))=Q(t), \quad t \geq T_{1} .
$$

Since $v(t)+P(t)>c+P(t)>0$, (9) has an eventually positive 
solution or, for $n$ odd, lim inf $r(t)>0$, a contradiction. $t \rightarrow \infty$

An analogous proof can be given if we start with an eventually negative solution of (2). This completes the proof.

REMARK. The above theorem is not covered by the results of Onose [5].

\section{References}

[1] F.V. Atkinson, "On second order differential inequalities", Proc. Roy. Soc. Edinburgh Sect. A 72 (1972/1974), 102-127 (1975).

[2] Athanassios G. Kartsatos, "On nth-order differential inequalities", J. Math. Anal. Appl. 52 (1975), 1-9.

[3] Athanassios G. Kartsatos, "Recent results on oscillation of solutions of forced and perturbed nonlinear differential equations of even order", Stability of dynomical systems (NSF-CMBS Conference, Mississippi State University, 1975, to appear).

[4] И.Т. Нигурадэе [I.T. Kiguradze], "О нолеблемости решений уравнения $\frac{d^{m} u}{d t^{m}}+a(t)|u|^{n} \operatorname{sign} u=0 "$ [On the oscillation of solutions of the equation $\frac{d^{m} u}{d t^{m}}+a(t)|u|^{n}$ sign $u=0$ ], Mat. Sb. 65 (107) (1964), 172-187.

[5] Hiroshi Onose, "A comparison theorem and the forced oscillation", Bull. Austral. Math. Soc. 13 (1975), 13-19.

Department of Mathematics, University of South Florida,

Tampa,

Florida, USA;

Department of Mathematics, Ibaraki University, Mi to, Japan. 